\title{
Apparatus 4 Flow Through Cell: Some Thoughts on Operational Characteristics
}

Will Brown

USP, Rockville, MD

email:web@usp.org

$\mathrm{T}$ he dissolution process is limited by the approach of the resulting solution to saturation. The establishment of sink conditions is a basic requirement of a dissolution method, ensuring that the observed dissolution rate reflects the behavior of the sample and not the solubility of the substance. With the paddle or basket apparatus, the volume is limited to the capacity of compendial vessels, 1-,2-, or 4- L. The development of the flow through cell came about as an attempt to provide a controlled hydrodynamic environment and renewable solvent stream to the sample. The literature reports that as early as 1957, the FDA had developed a flowing medium dissolution apparatus (1). At the time, solubility limitations in the existing constant-volume, or beaker, methods were a concern as efforts were directed to the dissolution properties of less soluble drug products. Various researchers worked with a tubular vertical cell that was eventually commercialized and described in the literature $(2,3,4,5$, and 6$)$

My experience with the flow through cell system began in 1990 when the USP Drug Research and Testing Laboratory began an evaluation of the apparatus for possible inclusion in the general test chapter, Drug Release $\langle 724\rangle$, which gives guidance on the performance of dissolution testing on modified release dosage forms. At that time, an article was published in Pharmacopeial Forum giving a description of the system and some data from studies using USP calibrator tablets (7). Erenfried Wirbitzki and Beda Fischer, two talented European scientists trained me at the USP on the operation of the apparatus, giving me the impression that the flow through cell attracted more interest in Europe than it had in this country.

\section{Description and Harmonization}

The compendial description of the device is in a good state of harmonization between the information given in the EP,JP, and USP (8). The basic cell design is of a vertical cylinder with a conical portion communicating between it and the delivery tubing. The flow is in an upward direction. The potential for turbulence with the outflow of a small tube into a large tube has been noted (9). The $40^{\circ}$ conical section of the cell compensates in part. Filling it with 1-mm glass beads has been said to produce laminar flow conditions ( 1 , 7,10 , and 11). Two compendial cell diameters, 12 - and 22.6$\mathrm{mm}$, are available. The peculiar number, $22.6 \mathrm{~mm}$, is designed to provide a cross-sectional area for that cell of $4.01 \mathrm{~cm}^{2} .12 \mathrm{~mm}$ gives approximately half that area or 1.13 $\mathrm{cm}^{2}$. Early researchers recognized that the dissolution rate for samples would be related more to the linear flow rate than to other measures (2). Glass beads of approximately 1$\mathrm{mm}$ diameter have a loosely tapped density of $1.52 \mathrm{~g}$ per $\mathrm{mL}$; the literature contains various recommended masses to be added to fill the cone in 'laminar' operation. The volume of the cone is approximately $0.6 \mathrm{~cm}^{3}$ for the $12-\mathrm{mm}$ cell and $4.2 \mathrm{~cm}^{3}$ for the larger cell. Thus $0.9 \mathrm{~g}$ and $6.4 \mathrm{~g}$ of glass beads will fill the 12 and $22.6 \mathrm{~mm}$ cells, respectively. In operation a 5 -mm bead is placed at the cone apex, preventing material from descending into the inlet tubing.

A number of other designs are available for specialized techniques associated with performance testing of suppositories, powders, and implants (12).

\section{Operational Considerations}

Open versus closed configuration. In operation, the flowing medium is pumped past the sample in the cell. The medium used can either be delivered fresh or can be re-circulated by the pump. Where the medium is delivered fresh and the eluate removed, the system is termed open. The medium from the open system can be collected as the entire outflow over the sampling interval. When this is done, the average concentration representing the cumulative mass dissolved in the total volume consumed is measured from the analytically-determined concentration and the volume collected. This average concentration is divided by the number of minutes elapsed to give an estimate of the release rate over the interval. This approach is designated cumulative. If this technique is followed carefully with a gravimetric determination of the volume collected, not only is an accurate measure of the flow rate but also an accurate measure of the release rate is possible. This should be the basis when validating alternative approaches to flow rate control and reporting. Reducing the sampling interval over a decreasing time period allows an approach to the estimate of instantaneous release rate. In the limit where the outflow transits the beampath of a fiberoptic probe, is transferred to a small volume autosampler vial or is passed through a spectrophotometer cuvette, the actual sampling interval is just the time taken in the immediate process. Thus the estimated release rate approaches an instantaneous value. In all open-loop systems, the solution concentration represents just the amount released over the interval collected. Accounting of the various data is needed to determine the percentage of the labeled dose dissolved over the sum of all intervals.

The closed-loop configuration uses a constant volume of medium in the procedure. The medium passes the sample and is returned by the pump to the flow-through cell and sample. A reservoir is placed in the line allowing the 


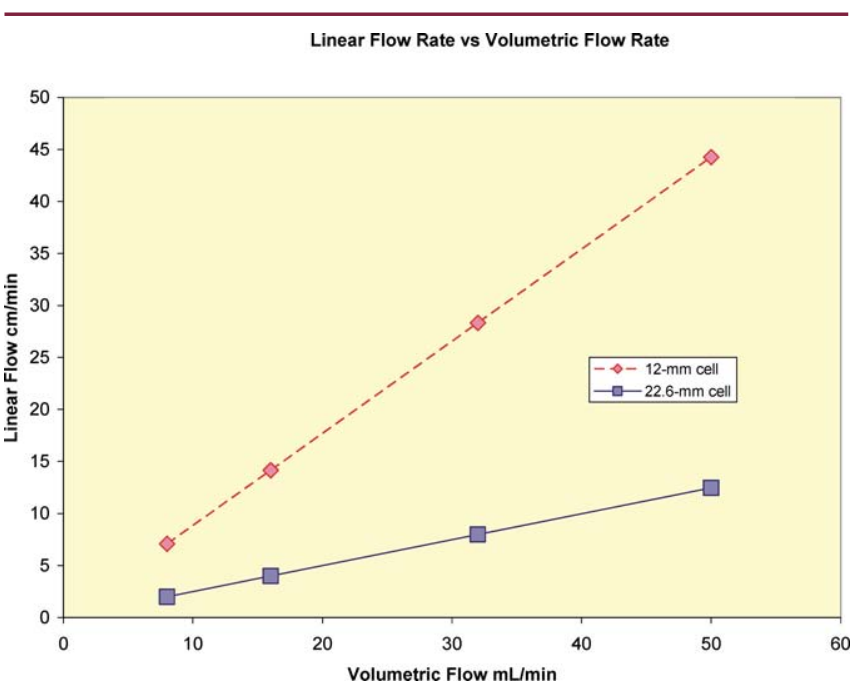

Figure 1. Comparison of linear flow rates for the 22.6- and 12-mm flowthrough cells at identical volumetric flow rates.

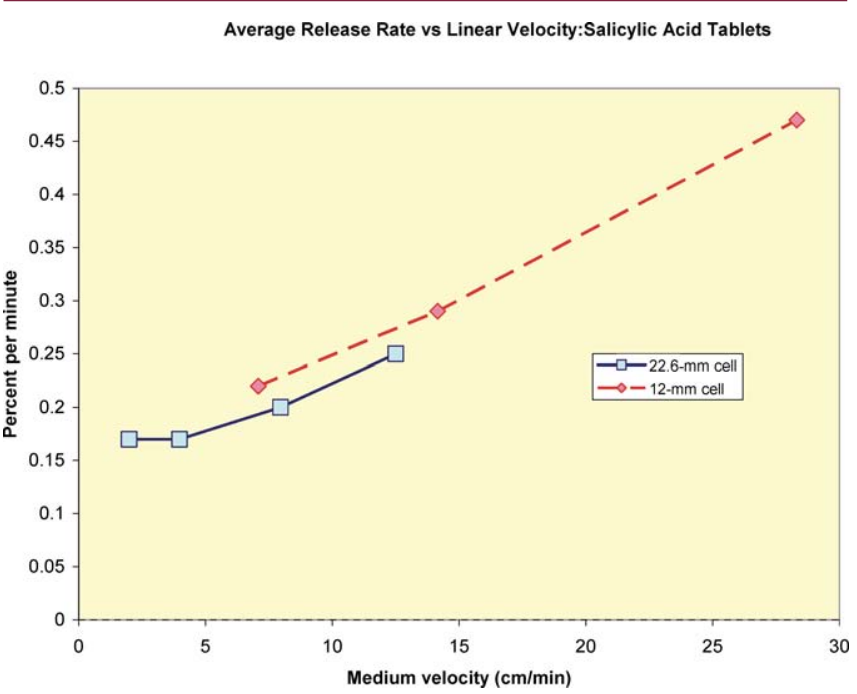

Figure 2. Salicylic Acid Non-Disintegrating Calibrator Tablets, Lot M 0.05 M phosphate buffer, $\mathrm{pH} 7.4$, tablets placed horizontally on the mid-cell clip in the 'laminar' configuration. Average amount dissolved from 30 to $60 \mathrm{~min}$ utes plotted versus linear flow rate for the 12- and 22.6- $\mathrm{mm}$ cells.

medium to be stirred, heated and sampled. The concentration of the drug increases in the medium over time; so this option is best taken when solubility is less of an issue than other concerns, such as the limit of quantification. This fixed-volume approach is familiar to those of us who typically use the paddle and basket. By determining the concentration of analyte and the volume in the system, the cumulative release can be calculated directly. The total volume of the system (tubing, flow-through cell, and reservoir) is most evidently limited by the reservoir. A volume less than about 20-30 mL may be impractical with current compendial design. In common with the open-loop configuration, a portion of the outflow can be removed from the stream or reservoir and pumped through a spectrophotometer cuvette past a fiber optic probe or collected to a vial for ultimate injection into a chromatograph.
Average Release Rate vs Linear Velocity:Prednisone Tablets

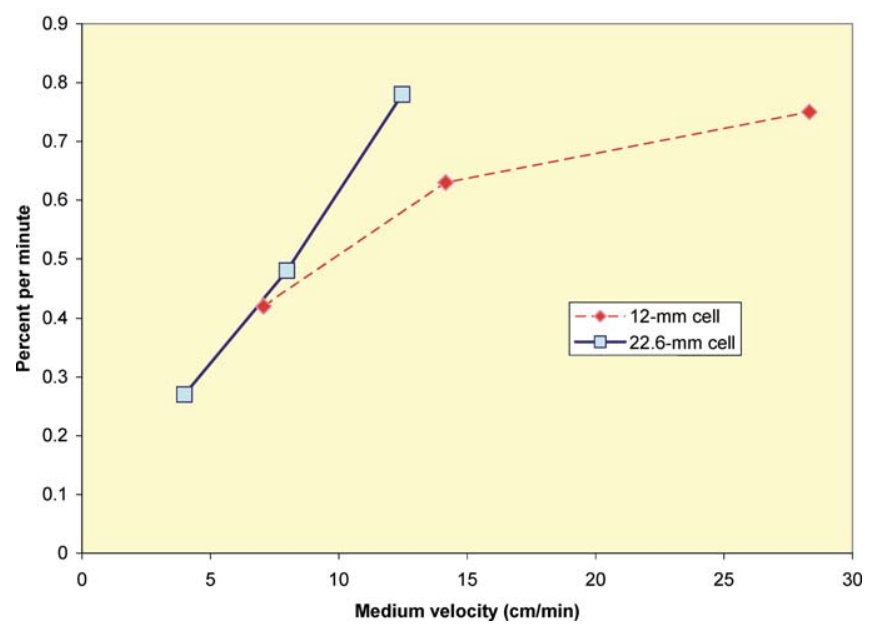

Figure 3. Prednisone Disintegrating Calibrator Tablets, Lot $K$ water, tablets placed horizontally on the mid-cell clip in the 'laminar' configuration. Average amount dissolved from 30 to 60 minutes plotted versus linear flow rate for the 12- and 22.6- $\mathrm{mm}$ cells dissolution rate averaged over dissolution testing for a thirty-minute sample collected at equivalent linear flow rates.

Small hold-up volume. An advantage of the flow-through cell system is the small volume in which the actual dissolution takes place. In contrast to the paddle or basket techniques, the volume of the 12-mm cell without glass beads is slightly over $8 \mathrm{~mL}$ and the volume of the $22.6-\mathrm{mm}$ cell without glass beads is nearly $19 \mathrm{~mL}$. The volume of the glass beads, coupled with any volume accounted for by the inlet and outlet tubing and filter chamber, allows dissolution to be carried out for samples with low drug loading and release over extended periods. At a nominal flow of $8 \mathrm{~mL}$ per minute the 12-mm cell body can be filled in one minute. In the closed-loop configuration recycling the dissolution medium through the cell amplifies this advantage with total volume reduced to an absolute minimum.

Cross-sectional area and flow rate. The effect of flowing medium on the dissolution rate has been well established. For samples placed in the flow cell, the linear velocity of the medium is particularly important. Thus the effect of the different compendial cells, 12 - and $22.6 \mathrm{~mm}$, should be a consideration. The linear velocity of the medium is related to the volumetric rate through the cross-sectional area of the two cells. The calculated linear velocity is inversely related through the ratio of the squared radii. One expects, at a given volumetric flow, the linear velocity of the smaller cell to be greater than that for the large cell by a factor of $3.5\left[(11.3 / 6)^{2}\right]$. The nearly four-fold difference in performance between the two compendial cell designs is shown in the comparison of linear flow at a given volumetric setting in Figure 1. Figure 2 gives a comparison of the average dissolution rate for the USP Salicylic Acid calibrator tablets at equivalent linear flow rates for the large and small flow-through cells (13). It should be noted from this data that linear flow rate may provide a basis of comparison between the two cells for a non-disintegrating, 
non-dispersed sample. From the data, it would appear that the dissolution rate of a non-dispersed sample might be more easily manipulated with the 12-mm cell. Figure 3 gives the relationship between the dissolution rate for the two cell sizes for a dispersed sample. Here the greater area available in the larger cell seems to outweigh the more accessible linear flow rates provided by the smaller cell (13).

Local environments in the cell. The flow-through cell can present several standard environments to the sample. When operated without glass beads in the entry cone, the condition is termed, "turbulent". With glass beads, laminar flow conditions are said to prevail. The sample can be placed within the glass-bead bed, or upon it, or with a wire holder provided to facilitate placement of the sample approximately midway along the cylindrical section. Each of the possible configurations can be associated with different resulting drug-release rates. The release of salicylic acid from USP calibrator tablets was found to be highest when the tablet was buried in the glass beads, followed by placement on top of the bead bed. The lowest release rate at a given flow was with the tablet secured to the mid-cell wire holder (14). For a disintegrating dosage form, initial placement on the holder under turbulent conditions gave an equivalent rate of release to that obtained with the tablet placed in the cone at the start of the experiment. It would seem that the tablet particles will move to the same position in the cell upon disintegration.

Sink conditions? Local conditions can cause saturation to occur as reported by Posti (15). Samples of acetaminophen (paracetamol) particles were subjected to dissolution commingled with glass beads in the $22.6-\mathrm{mm}$ flow-through cell. It should be noted that the free volume in packed 1-mm beads would be about $40 \%$ of the volume in which they are contained. The dissolution rate for a ten-fold larger sample of the fine powder did not increase over that for the smaller sample mass of the same powder and, in fact, was diminished. The authors concluded that the increased surface area available in the larger sample enabled the saturation of the medium to occur; thus, counter-intuitively, the dissolution was inhibited.

\section{Conclusion}

The flow-through cell was developed to answer some deficiencies perceived in other compendial techniques. Guidance is given in major pharmacopoeias. A variety of analytical techniques that can be applied to a variety of samples are available. Sampling can represent almost instantaneous changes. The larger flow cell may offer a less aggressive environment than its smaller sister. The flowthrough cell continues to be worthy of study and use as a performance technique.

\section{References}

1. F Langenbucher, D Benz,W Kurth, H Moller, M Otz, Standardized Flow-cell Method as an Alternative to Existing Pharmacopoeial Dissolution Testing, Pharm Ind 51 (11):1276-1281, 1989

2. F Langenbucher, In Vitro Assessment of Dissolution
Kinetics: Description and Evaluation of a Column-type Method, J Pharm Sci, 58(10):1265-1272, 1969

3. DC Baun, GCWalker, Apparatus for Determining the Rate of Drug Release from Solid Dosage Forms, J Pharm Sci 58(5):611-616, 1969

4. J ETingstad, S Riegelman, Dissolution Rate Studies I: Design and Evaluation of a Continuous Flow Apparatus, J Pharm Sci 59(5):692-696, 1970

5. C Cakiryildiz, P J Mehta, W Rahmen, D Shoenleber, Dissolution Studies with a Multichannel Continuous-Flow Apparatus, J Pharm Sci 64(10): 1692-1697, 1975

6. F Langenbucher, $\mathrm{H}$ Rettig, Dissolution rate Testing with the Column Method:Methodology and Results, Drug Dev Ind Pharm 3(3):241-262, 1977

7. M Nicklasson, F Langenbucher, Description and Evaluation of the Flow Cell Dissolution Apparatus as an Alternative Test Method for Drug Release, Pharm Forum 16(3):532-540, 1990

8. <711> Dissolution, Pharm Forum 31(2):360-367, 2005

9. SR Cammarn, A Sakr, Predicting dissolution via hydrodynamics: salicylic acid tablets in flow through cell dissolution, Int J Pharm 201:199-209,2000

10. G H Zhang, W A Vadino, TT Yang, W P Cho, I A Chaudry, Evaluation of the flow-through cell dissolution apparatus: Effects of flow rate and tablet position on drug release from different type of tablets, Drug Dev Ind Pharm 20(13):2063-2078, 1994

11. N Bielen, Performance of USP calibrator tablets in flowthrough cell apparatus, Int J Pharm 233: 1234-129, 2002

12. H Moller, E Wirbitzki, Regulatory aspects of modified release dosage forms: special cases of dissolution testing using the flow-through system, Boll. Chim. Farmaceutico 132(4):105-115, 1993

13. W E Brown, USP Apparatus 4 Calibration:a chemist's view, Presentation at Dissolution workshop, AAPS Annual Meeting 1996

14. M Morihara, N Aoyagi, N Kaniwa, N Katori, S Kojim, Hydrodynamic flows around tablets in different pharmacopeial dissolution tests, Drug Dev Ind Pharm 28(6):655-662,2002

15. J Posti, P P Spieser, Sink conditions in the flow-through cell during dissolution, Int J Pharm 5: 101-107, 1980 